\title{
Endoscopic Transpapillary Gallbladder Drainage in a Patient with Billroth-II Reconstruction
}

\author{
Yuichi Takano, Jun Noda, Masataka Yamawaki, Tetsushi Azami, Takahiro Kobayashi, \\ Fumitaka Niiya, Naotaka Maruoka and Masatsugu Nagahama
}

\begin{abstract}
:
Endoscopic transpapillary gallbladder drainage (ETGBD) is an established procedure and is listed in the Tokyo guidelines 2018. Although there are many reports of ETGBD in normal anatomy cases, it is rarely performed for patients with a surgically altered anatomy. We herein report the case of a patient who underwent ETGBD with Billroth-II reconstruction. ETGBD could be an effective treatment option even for patients with a surgically altered anatomy.
\end{abstract}

Key words: Endoscopic transpapillary gallbladder drainage, acute cholecystitis, surgically altered anatomy

(Intern Med 60: 2613-2615, 2021)

(DOI: 10.2169/internalmedicine.6964-20)

\section{Introduction}

The accepted curative treatment for acute cholecystitis is surgery (cholecystectomy) (1). For patients in which surgery would present a high risk or from whom consent cannot be obtained, percutaneous transhepatic gallbladder drainage (PTGBD), endoscopic transpapillary gallbladder drainage (ETGBD), and endoscopic ultrasound-guided gallbladder drainage (EUS-GBD) are suitable. ETGBD is an established procedure and is listed in the Tokyo guidelines 2018 (TG 18) (2). Although there have been many reports of ETGBD in normal anatomy cases (3-5), it is rarely performed for patients with a surgically altered anatomy. We herein report the case of a patient who underwent ETGBD with BillrothIreconstruction.

\section{Case Report}

The patient was an 83-year-old man. Due to a gastric ulcer, he had undergone distal gastrectomy and Billroth-II reconstruction 50 years earlier. There was nothing special to note in his family history. He had visited the emergency department for epigastric pain 3 days earlier. His vital signs were a body temperature of $38.2^{\circ} \mathrm{C}$, blood pressure of $145 /$ $70 \mathrm{mmHg}$, and heart rate of $103 / \mathrm{min}$. Tenderness was ob- served in the epigastric region, and Murphy's sign was positive. Blood tests indicated high inflammatory responses, as shown by a white blood cell count of $14,000 / \mu \mathrm{L}$ and Creactive protein concentration of $10.8 \mathrm{mg} / \mathrm{dL}$, but his hepatobiliary enzyme levels were normal. Abdominal computed tomography showed numerous stones inside a swollen gallbladder accompanied by fat stranding (Fig. 1). A diagnosis of acute cholecystitis GradeII was made according to the TG 18. PTGBD was performed urgently, and his cholecystitis improved.

Elective surgery was recommended, but the patient and his family refused. Therefore, we decided to remove the PTGBD and clamped it. However, 2 days later, abdominal pain recurred, and he was diagnosed with recurrent cholecystitis. The symptoms improved after reopening the PTGBD. We then decided to perform ETGBD to remove the PTGBD.

The papilla of Vater was reached by single-balloon endoscopy (SIF-H290S; Olympus Medical Systems, Tokyo, Japan). Cholangiography revealed numerous stones filling the gallbladder and cystic duct. A 0.025-inch guidewire (Visiglide2; Olympus) was deeply passed into the gallbladder. A 5-Fr transpapillary gallbladder stent (IYO-stent ${ }^{\mathrm{TM}}$; Gadelius, Tokyo, Japan) was placed (Fig. 2, 3). There were no complications associated with the procedure. The PTGBD was removed, and the patient was thereafter dis- 
charged. At 6 months after the procedure, no recurrence of acute cholecystitis was observed.

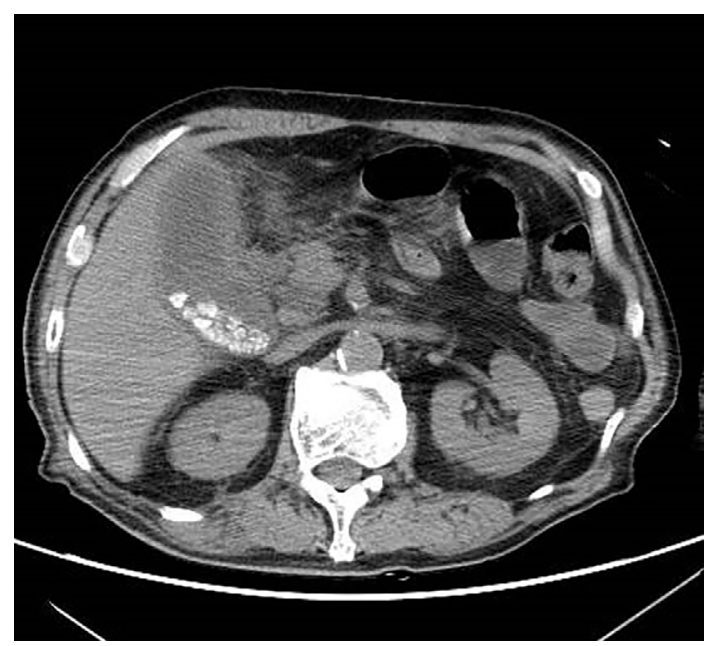

Figure 1. Abdominal computed tomography showed numerous stones inside a swollen gallbladder accompanied by fat stranding.

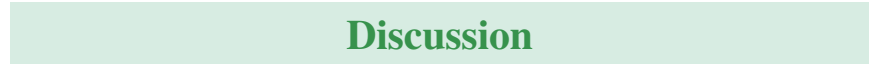

Currently, ETGBD is widely performed for acute cholecystitis (2). Good indications for ETGBD include a bleeding tendency, massive ascites, and Chilaiditi syndrome (5). In a recent meta-analysis, the technical success of ETGBD was $83 \%$, clinical success was $88.1 \%$, and adverse events frequency was $9.6 \%$ (6). Attention should be paid to complications such as pancreatitis and perforation of the cystic duct. It is not a procedure that is easy to perform and it thus requires advanced techniques. Breaking through the cystic duct with a guidewire or inserting a stent requires a high degree of skill. The TG18 states that ETGBD may be considered in institutions with pancreaticobiliary endoscopy experts, but PTGBD should be the standard drainage in institutions where such experts are not available $(1,2)$.

EUS-GBD is an alternative drainage method that has attracted attention in recent years because it offers one-step internal drainage with a low risk of developing pancreatitis (7). However, serious complications, such as biliary peri-
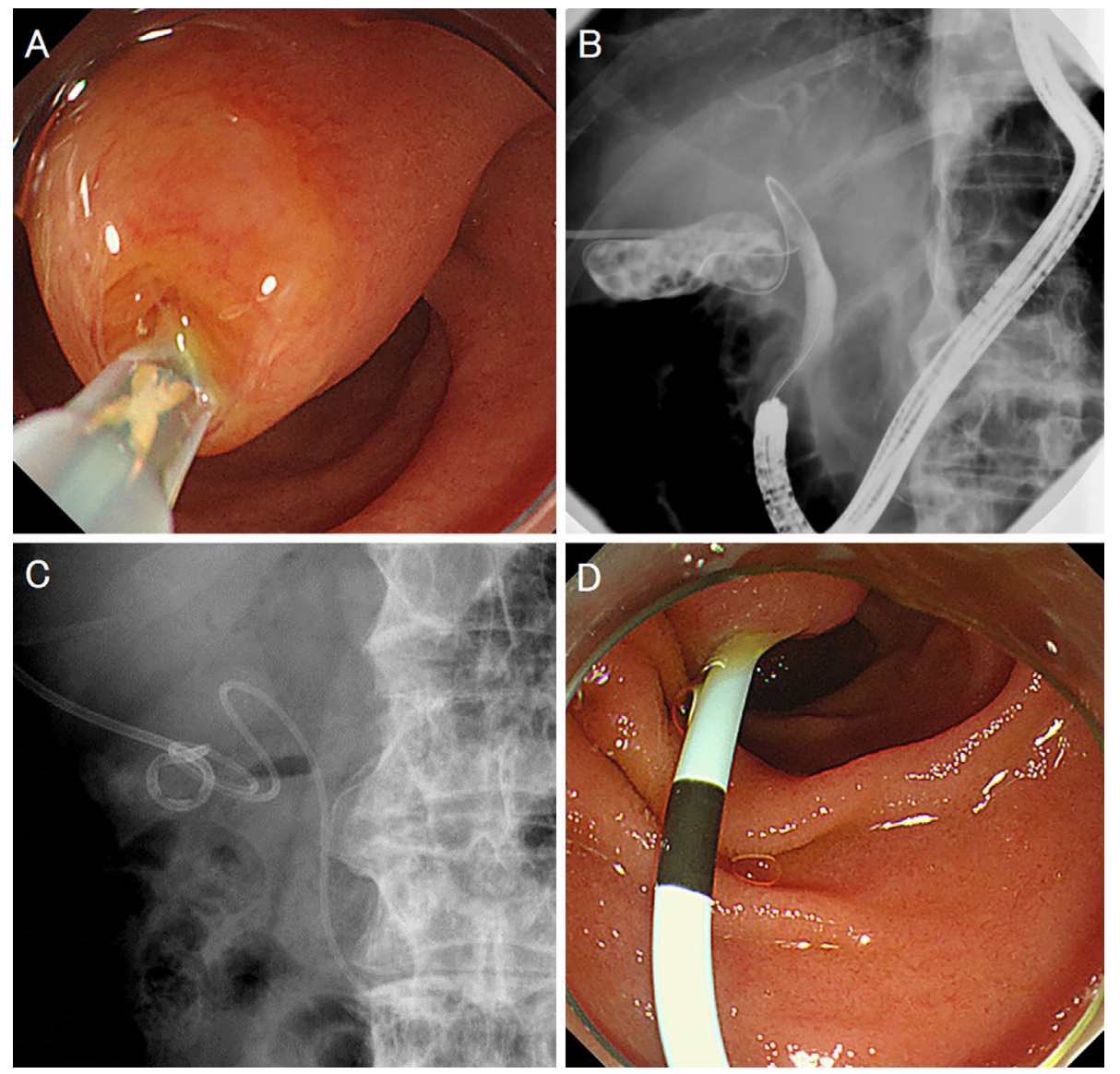

Figure 2. A: Endoscopic retrograde cholangiography was performed using a single balloon endoscope. Deep bile duct cannulation was achieved using a conventional catheter. B: Cholangiography revealed numerous stones that filled the cystic duct and gallbladder. Percutaneous transhepatic gallbladder drainage was already inserted. A guidewire was deeply passed into the gallbladder, and a 5-Fr transpapillary gallbladder stent was placed. C: A plain abdominal radiograph obtained on the day after the procedure was performed. Both percutaneous transhepatic gallbladder drainage and transpapillary gallbladder drainage are placed. D: An indwelling transpapillary gallbladder stent. 


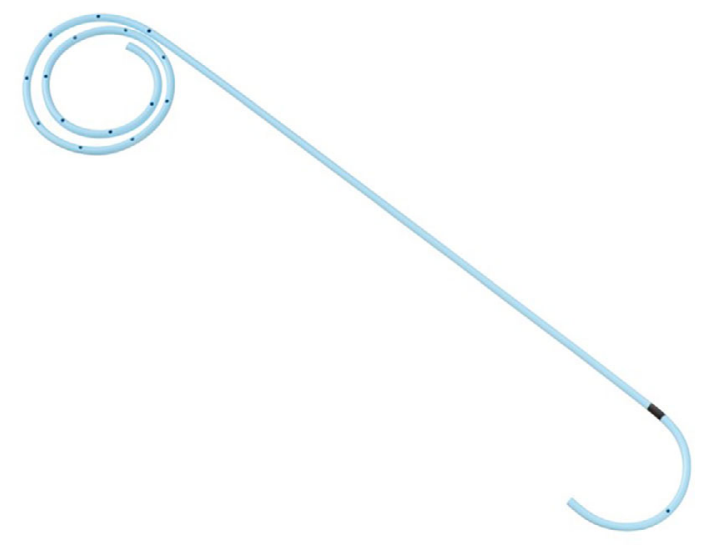

Figure 3. A 5-Fr transpapillary gallbladder stent (IYOstent $^{\mathrm{TM}}$, Gadelius, Tokyo, Japan).

tonitis and intraperitoneal migration of the stent, can occur. In patients with a surgically altered anatomy, some distance between the digestive tract and gallbladder can be created, and EUS-GBD is not possible in all patients.

In recent years, with the advent of balloon endoscopes, the use of endoscopic retrograde cholangiopancreatography (ERCP) in surgically altered anatomies has shown great progress (8). Good results similar to those forpatients with a normal anatomy have been reported for the treatment of common bile duct stones and bile duct stricture. However, ETGBD is rarely performed in patients with a surgically altered anatomy partly because of the difficult and complex procedures and uncertain outcomes.

A duodenoscope and an upper gastrointestinal endoscope are also used in ERCP in cases of Billroth II reconstruction. However, in patients with long afferent loops or severe adhesions, a single-balloon endoscope is more likely to reach the Vater papilla. For this reason, the single-balloon endoscope is the first choice in ERCP in cases of Billroth II reconstruction at our department. When performing recently developed single-balloon endoscopy (SIF-H290S, Olympus) which had a $152-\mathrm{cm}$ working length and a 3.2-mm working channel, many of the devices used in conventional ERCP can be used. In our case, it was possible to smoothly place a transpapillary gallbladder stent. The stent had a small diameter of 5-Fr, it was easy to pass through desired openings, and can be inserted without problems even in patient with strong cystic duct flexion. In addition, the stent length is one size $(32 \mathrm{~cm})$, and the stent length need not be ad- justed in individual cases. ETGBD could be an effective treatment option even for patients with a surgically altered anatomy.

In this case, a transpapillary gallbladder stent was to be permanently placed. When cholecystitis relapsed as a result of stent occlusion, we should first consider the endoscopic replacement of the gallbladder stent. PTGBD is also an option when the patient's general condition is poor (e.g., sepsis or shock is present) and such endoscopic procedures are associated with a high risk of complications.

The authors state that they have no Conflict of Interest (COI).

\section{References}

1. Okamoto K, Suzuki K, Takada T, et al. Tokyo Guidelines 2018: flowchart for the management of acute cholecystitis. J Hepatobiliary Pancreat Sci 25: 55-72, 2018.

2. Mori Y, Itoi T, Baron TH, et al. Tokyo Guidelines 2018: management strategies for gallbladder drainage in patients with acute cholecystitis (with videos). J Hepatobiliary Pancreat Sci 25: 87-95, 2018.

3. Ogawa O, Yoshikumi H, Maruoka N, et al. Predicting the success of endoscopic transpapillary gallbladder drainage for patients with acute cholecystitis during pretreatment evaluation. Can J Gastroenterol 22: 681-685, 2008.

4. Yane K, Maguchi H, Katanuma A, et al. Feasibility, efficacy, and predictive factors for the technical success of endoscopic nasogallbladder drainage: a prospective study. Gut Liver 9: 239-246, 2015.

5. Itoi T, Sofuni A, Itokawa F, et al. Endoscopic transpapillary gallbladder drainage in patients with acute cholecystitis in whom percutaneous transhepatic approach is contraindicated or anatomically impossible (with video). Gastrointest Endosc 68: 455-460, 2008.

6. Mohan BP, Khan SR, Trakroo S, et al. Endoscopic ultrasoundguided gallbladder drainage, transpapillary drainage, or percutaneous drainage in high risk acute cholecystitis patients: a systematic review and comparative meta-analysis. Endoscopy 52: 96-106, 2020.

7. Itoi T, Itokawa F, Kurihara T. Endoscopic ultrasonography-guided gallbladder drainage: actual technical presentations and review of the literature (with videos). J Hepatobiliary Pancreat Sci 18: 282286, 2011.

8. Katanuma A, Yane K, Osanai M, et al. Endoscopic retrograde cholangiopancreatography in patients with surgically altered anatomy using balloon-assisted enteroscope. Clin J Gastroenterol 7: 283289, 2014.

The Internal Medicine is an Open Access journal distributed under the Creative Commons Attribution-NonCommercial-NoDerivatives 4.0 International License. To view the details of this license, please visit (https://creativecommons.org/licenses/ by-nc-nd/4.0/).

\section{(C) 2021 The Japanese Society of Internal Medicine} Intern Med 60: 2613-2615, 2021 